Mens

Revue d'histoire intellectuelle et culturelle

\title{
Ramsay Cook (1931-2016) : hommage et témoignages
}

\section{Sylvie Beaudreau et Xavier Gélinas}

Volume 18, numéro 1, automne 2017

URI : https://id.erudit.org/iderudit/1062930ar

DOI : https://doi.org/10.7202/1062930ar

Aller au sommaire du numéro

Éditeur(s)

Centre de recherche en civilisation canadienne-française

ISSN

1492-8647 (imprimé)

1927-9299 (numérique)

Découvrir la revue

Citer ce document

Beaudreau, S. \& Gélinas, X. (2017). Ramsay Cook (1931-2016) : hommage et

témoignages. Mens, 18(1), 7-20. https://doi.org/10.7202/1062930ar d'utilisation que vous pouvez consulter en ligne.

https://apropos.erudit.org/fr/usagers/politique-dutilisation/ 


\title{
Ramsay Cook (1931-2016) : hommage et témoignages
}

\author{
Sylvie Beaudreau, SUNY Plattsburgh \\ Xavier Gélinas, Musée canadien de l'histoire
}

Le 14 juillet 2016 s'éteignait Ramsay Cook, longtemps professeur à l'Université de Toronto (1958-1968), puis à l'Université York (19681996), auteur de dizaines d'ouvrages et d'articles, superviseur de 39 thèses doctorales, directeur général du Dictionnaire biographique $d u$ Canada pendant 17 ans. En publiant ce bref texte, deux de ses anciens étudiants souhaitent saluer la mémoire d'un des historiens les plus renommés du pays et présenter les grandes lignes de son œuvre.

\section{L'homme et l'ouvre}

Né dans la petite ville d'Alameda, en Saskatchewan, le 28 novembre 1931, d'un père pasteur de l'Église unie qui changea souvent de paroisse et de ville, Ramsay Cook fait ses études universitaires au United College (devenu l'Université de Winnipeg), puis à Queen's, sous la supervision d'Arthur Lower, et enfin à l'Université de Toronto sous l'égide de Donald Creighton, alors au faîte du panthéon des historiens anglo-canadiens. Publiée en 1963, sa thèse s'inscrit dans le sillon de l'histoire politique classique. Elle porte sur le journaliste John W. Dafoe, du Winnipeg Free Press, qui a fait la pluie et le beau temps dans le monde politique et, singulièrement, dans l'Ouest canadien et au Parti libéral, pendant quatre décennies ${ }^{1}$.

\footnotetext{
1 Ramsay Cook, The Politics of John W. Dafoe and The Free Press, Toronto, University of Toronto Press, 1963.
} 
Dès le début des années 1960, l'intérêt de Cook est aiguisé par les soubresauts qui agitent le Québec. Commence alors une décennie très féconde où, par des études savantes et des articles engagés, il s'efforce de mieux comprendre la situation, de s'en faire l'interprète auprès des milieux intellectuels et politiques du Canada anglais et, inversement, de présenter au Québec la perspective d'un AngloCanadien attentif. C'est que, de surcroît, le " reste du Canada " traverse lui aussi son " autre Révolution tranquille », pour reprendre l'expression de José Igartua ${ }^{2}$; Cook se penche également sur ce processus de redéfinition. Il devient paradoxalement l'une des figures de proue de ce néonationalisme canadien émergent, en proposant de mettre un bémol sur les filiations historico-coloniales et d'élargir plutôt la définition du canadianisme aux «identités limitées » ou partielles que constituent les classes sociales, l'appartenance régionale et les divers groupes ethniques. À titre de citoyen, il déserte avec fracas le Nouveau Parti démocratique, où se portent ses penchants naturels, lorsque ce dernier épouse la thèse des " deux nations ». Il appuie avec enthousiasme l'irruption sur la scène politique de Pierre Elliott Trudeau, qui lui semble cristalliser ses idées.

La production de Cook durant les années 1960 est saisissante. On lui doit notamment, en 1966, Canada and the French-Canadian Question, recueil d'études et d'essais dont une traduction paraît sous le titre Le sphinx parle français ${ }^{3}$. Il faut dire que Cook commence à se tailler une réputation enviable, dans les cercles intellectuels québécois, comme interlocuteur anglophone. Cet accueil est largement dû à la place que Claude Ryan, autre esprit féru de dialogue, lui accorde

\footnotetext{
2 José Igartua, The Other Quiet Revolution: National Identities in English Canada, 1945-71, Vancouver, University of British Columbia Press, 2006. Voir aussi C. P. Champion, The Strange Demise of British Canada: The Liberals and Canadian Nationalism, 1964-1968, Montréal, McGill-Queen’s University Press, 2010.

3 Ramsay Cook, Canada and the French-Canadian Question, Toronto, Macmillan of Canada, 1966; Le sphinx parle français : un Canadien anglais sinterroge sur le problème québécois, trad. de François Rinfret, Montréal, Éditions HMH, 1966, coll. «Aujourd'hui».
} 
dans les pages du Devoir dès $1964^{4}$. La décennie se clôt pour lui, en 1969, par la publication de deux ouvrages. Il s'agit, d'une part, d'une étude commandée par la Commission sur le bilinguisme et le biculturalisme, L'autonomie provinciale, les droits des minorités et la théorie du pacte, 1867-19215. D'autre part, Cook a la bonne idée de colliger des textes clés de l'histoire du nationalisme canadien-français, de François-Xavier Garneau au jeune Jean-Marc Piotte de la revue Parti pris, à l'intention du public anglophone : ce sera French-Canadian Nationalism: An Anthology ${ }^{6}$. En 1971 parait The Maple LeafForever ${ }^{7}$, autre recueil d'essais parus dans les années précédentes et qui sonde la psyché nationale canadienne dans son ensemble.

Les années 1970 marquent un élargissement de ses champs d'intérêt. Sans abandonner la riche "French Canadian question", Cook dirige son attention sur d'autres domaines, sur ces identités limitées dont il martelait l'importance. Cela donnera lieu à des publications qui témoignent de sa fécondité intellectuelle et de son refus de se laisser enfermer dans un territoire restreint. On le voit ainsi se pencher sur l'histoire des femmes, par exemple, sur l'histoire de l'art et sur les rapports complexes entre philosophie, religion et action sociale. Son livre, The Regenerators (1985), sera couronné de prix, dont celui du gouverneur général ${ }^{8}$. S'il propose des focalisations nouvelles, Cook ne néglige pas pour autant le grand angle. Avec Craig Brown, il publie en 1974 une synthèse des années 1896-1921, qui

${ }^{4}$ Voir Michael Gauvreau, The Hand of God: Claude Ryan and the Fate of Canadian Liberalism, 1925-1971, Montréal, McGill-Queen's University Press, 2017, p. 370, 384, 418-419, 486, 500 et 540.

5 Ramsay Cook, L'autonomie provinciale, les droits des minorités et la théorie du pacte, 1867-1921, Ottawa, Imprimeur de la Reine, 1969, coll. «Études de la Commission royale d'enquête sur le bilinguisme et le biculturalisme ". La version originale s'intitule Provincial Autonomy, Minority Rights and the Compact Theory, 1867-1921.

${ }^{6}$ Ramsay Cook, French-Canadian Nationalism: An Anthology, Toronto, Macmillan of Canada, 1969.

7 Ramsay Cook, The Maple Leaf Forever: Essays on Nationalism and Politics in Canada, Toronto, Macmillan of Canada, 1971.

8 Ramsay Cook, The Regenerators: Social Criticism in Late Victorian English Canada, Toronto, University of Toronto Press, 1985. 
montre la polyvalence de son approche historienne. Trois ans plus tard, avec John Ricker et John Saywell, il publie une mise à jour d'un livre de poche couvrant toute l'histoire du pays, dont la première édition datait de 1963 et qui avait connu un grand succès de vente?. En 1989, autre grande étape du parcours de Cook, il devient directeur général du monumental Dictionnaire biographique du Canada, qui traverse des difficultés administratives. Cet homme sensible, ce socialdémocrate doit prendre des décisions difficiles, faire des coupures, tendre la sébile pour renflouer l'entreprise; il réussit à maintenir le navire $\mathrm{DBC}$ à flot et à l'adapter aux nouvelles réalités numériques. Sa retraite officielle de York en 1996 ne marque nullement la fin de son œuvre. Outre le fait qu'il continue de tenir les rênes du Dictionnaire jusqu'en 2006, il lance notamment, la même année, un ouvrage à la fois senti et réflexif sur son ami Trudeau ${ }^{10}$.

Les pages qui suivent, écrites à la première personne, proposent deux témoignages.

\section{Témoignage de Sylvie Beaudreau}

Au cours d'un séjour récent à Toronto pour le mariage de ma nièce, je me suis prise à penser à la trajectoire de ma carrière universitaire. À plus de 20 ans de distance, de Toronto et de mes études supérieures, il m'a semblé naturel de réfléchir à la bonne fortune qu'avait été le choix de l'Université York et d'un directeur comme Ramsay Cook.

Au début du programme, j'étais nettement désavantagée par ma maîtrise en études européennes et victoriennes parmi les autres doctorants, qui s'étaient spécialisés en histoire canadienne. J'avais donc beaucoup de rattrapage à faire. J'ai souhaité que ma méconnaissance

\footnotetext{
9 Ramsay Cook, John C. Ricker et John T. Saywell, Canada: A Modern Study, Toronto, Irwin Publishing, 1977. L’ouvrage a connu une traduction française tardive : Le Canada : étude moderne, trad. de Claude Romney, Toronto, Clarke, Irwin \& Company Limited, 1981.

${ }^{10}$ Ramsay Cook, The Teeth of Time: Remembering Pierre Elliott Trudeau, Montréal, McGill-Queen's University Press, 2006.
} 
des faits de l'histoire du Canada soit compensée par la richesse des concepts couverts dans d'autres domaines.

Étudier avec Ramsay Cook s'est révélé judicieux. J'avais présenté ma demande depuis Montréal, et je ne pouvais m’empêcher de penser que mon nom francophone et ma province d'origine susciteraient l'intérêt de la direction d'un programme offert dans le foyer intellectuel par excellence du Canada anglais. La capacité de lire, de parler et d'écrire en français ferait peut-être de moi la «Canadienne francophone de service ", mais peu m'importait. Du reste, j'avais la nette impression qu'un doctorat au Québec me transformerait en historienne du Québec. En Ontario, en revanche, j'étais certaine d'obtenir un véritable doctorat en histoire du Canada, qui engloberait le Québec. L'idée d'étudier l'intégralité du pays dans toute sa diversité me plaisait beaucoup et j'étais fin prête.

Autrement dit, en choisissant de rester à Montréal pour étudier l'histoire socioculturelle du Québec, je me serais probablement retrouvée parmi une forte cohorte de spécialistes du Québec. En optant pour Toronto, j'étais un peu livrée à moi-même, un peu seule dans mon camp. Une sorte d'oiseau exotique. York m'ayant offert bourses d'études et mandats d'assistante d'enseignement, le choix n'a pas été difficile.

Bonne décision, également, que celle d'avoir demandé à Ramsay Cook de diriger ma thèse. Ses travaux avaient surtout porté, jusquelà, sur le nationalisme québécois, examiné parfois avec sympathie, toujours avec érudition et souvent dans le contexte plus vaste d'un nationalisme pancanadien naissant. Je savais qu'il était parvenu à la maturité professionnelle au cours des années 1960, si fécondes sur le plan intellectuel, tandis que le Canada se dotait d'un nouveau drapeau, faisait la fête à Expo 67 et célébrait son centenaire. Années grisantes, s'il en fut. Cook semblait avoir capitalisé intellectuellement l'idée de répondre à la question contrariante que posait le Canada anglais pendant la Révolution tranquille : que veut donc le Québec? Si Cook n’a pas fourni de réponse définitive, il a prouvé qu'il était possible à un intellectuel anglophone canadien d'être sensible aux 
aspirations du Canada français et, plus tard, des Québécois. Il a trouvé sa voie en même temps que des lumières comme Leonard Cohen, Pierre Elliott Trudeau et Margaret Atwood. J'ai senti qu'il serait fascinant de travailler avec lui.

J'avais raison. Je me dois d'abord d'évoquer l'édition revue de Canada: A Modern Study, rédigée avec Saywell et Ricker (voir la note 9). Il s'agit d'un survol de l'histoire du Canada en édition de poche, illustré de caricatures anciennes, qui m'a aidée à comprendre rapidement la trame du Canada. Beaucoup de manuels ont été publiés et sont disparus depuis, mais le pratique petit livre de Cook et de ses collaborateurs m'a été particulièrement précieux. J'en ai d'ailleurs en ce moment même un exemplaire maintes fois relu à portée de main. Autre classique, méconnu, The Craft of History ${ }^{11}$, mince ouvrage de 1973 acheté au World's Biggest Bookstore. Issu d'une minisérie télévisée présentée au réseau anglais de Radio-Canada, le livre renferme une série d'entretiens avec trois grands historiens canadiens Arthur Lower, Donald Creighton et Michel Brunet - sous la direction de Ramsay et d'Eleanor Cook. Ce livre m'a incitée à considérer Cook comme une sorte de passeur entre ces historiens établis et moi-même. J'ai eu le sentiment que Cook avait été formé par un impressionnant groupe de penseurs. Ce qu'il disait d'eux semblait prestigieux. Un jour, pendant une entrevue qu'il donnait au réseau anglais de RadioCanada, je l'ai entendu formuler, d'une façon assez peu compliquée, l'idée que l'histoire du Canada était un champ d'études important, qui exposait les spécialistes à toutes les tendances et à tous les courants de l'histoire occidentale, unique en soi et certainement digne d'étude et de réflexion. Des mots qui m'ont encouragée pendant toute ma formation.

Nombre des écrits de Cook ont influencé ma perception du Canada. J'ai découvert un érudit anglo-canadien sympathique au Canada français, en mesure d'écrire des livres qui mettaient en évi-

11 Eleanor Cook (dir.), The Craft of History, avec une introduction de Ramsay Cook, Toronto, Canadian Broadcasting Corporation, 1973, coll. «Images of Canada ». 
dence la structure de l'histoire du Canada. L'ouvrage clé, pour moi, a été Canada and the French Canadian Question. Le chapitre intitulé "Quebec: The Ideology of Survival " me semblait suggérer que la métaphore de la survie (appliquée aussi de manière éblouissante par Margaret Atwood à la littérature canadienne) était le sésame qui permettait de comprendre un peuple dont l'identité n'est pas toujours facile à saisir. Cook y revient dans Maple Leaf Forever: Essays on Nationalism and Politics in Canada. À un chapitre intitulé "La Survivance French Canadian Style » reprenant certains de ses thèmes, il en a ajouté un autre : "La Survivance English Canadian Style ». Il y suggérait en résumé que, si le Canada français se sentait menacé du fait d'être une minorité catholique de langue française sur un continent essentiellement protestant et anglophone, les insécurités du Canada anglais tenaient à un sentiment de fragilité plus grand encore devant la menace continue des États-Unis, barrière des langues exceptée. Les nationalismes francophone et anglophone au Canada semblaient avoir davantage de quoi les unir que les séparer. Mais surtout, les écrits de Cook m'ont aidée à structurer ma pensée globale.

Dans French Canadian Nationalism: An Anthology, Cook a réuni des textes de nationalistes canadiens-français francophones marquants, fournissant aux étudiants une sorte d'introduction à un courant de pensée qui, à y regarder de plus près, était assez nuancé. De FrançoisXavier Garneau à Lionel Groulx et à Léon Dion, en effet, les visions ont divergé. Je n'oublierai jamais que, sur une étagère de son grand bureau plein de livres, au Collège Vanier, Cook avait posé un portrait de Lionel Groulx. Il m’a paru intéressant que Cook, né à Alameda, en Saskatchewan, ait considéré Groulx comme une sorte de maitre à penser. Il prouvait ainsi, à tout le moins, que non seulement il avait étudié le Canada français, mais qu'il avait admiré ses penseurs et s'était imprégné de son passé.

C'est en travaillant avec Cook que m'est venue l'idée d'écrire un article sur la réaction de Groulx à la Révolution tranquille du Québec, 
qui a été publié dans la Revue d'histoire de l'Amérique française ${ }^{12}$. Cette réalisation me semblait prouver qu'une chercheuse montréalaise pouvait faire des recherches et écrire un article de qualité sur l'histoire du Québec depuis Toronto. Étudier avec Cook à Toronto, c'était un peu comme franchir un pas optimiste vers la conviction que le Canada avait un sens. Tout cela se passait à la fin des années 1980 et au début des années 1990, en même temps que la débâcle du lac Meech, l'Accord de libre-échange canado-américain, l'accord de Charlottetown et le second référendum sur la souveraineté du Québec, au résultat si serré. Ces événements tumultueux ont alimenté nombre de nos discussions hors des salles de cours. Même s'il avait dépassé l'âge du jeune loup aux dents longues, notre professeur n'était pas pour autant devenu blasé face aux débats qui agitaient le Québec et, par ricochet, le Canada. Il s'est ainsi immiscé avec fougue dans la controverse dite Richler-Delisle sur l'antisémitisme dans la société canadienne-française de l'entre-deux-guerres ${ }^{13}$. Sans doute est-ce un cas où le citoyen engagé, l'homme entier, a pris le dessus sur l'observateur tolérant et équanime?

Mais Cook m'a offert plus qu'une compréhension du Canada. Son travail montrait que ses intérêts étaient catholiques, au sens ancien du terme, c'est-à-dire universels. Pendant mes études avec lui, il a écrit The Regenerators: Social Criticism in Late Victorian English Canada, qui allait être primé. Grâce à cet ouvrage et à bien d'autres choses, j'ai été exposée au victorianisme du Canada anglais, ce qui m'a permis d'approfondir les recherches que j'avais faites pour ma maîtrise en études victoriennes. Cette fascination de toujours a pris une nouvelle orientation avec Cook. J'ai compris combien ce domaine pouvait être richement évocateur si on l'appliquait au Canada de

12 Sylvie Beaudreau, «Déconstruire le rêve de nation : Lionel Groulx et la Révolution tranquille ", Revue d'histoire de l'Amérique française, vol. 56, nº 1 (été 2002), p. 29-61.

13 Ramsay Cook, "The Remembrance of All Things Past ", préface à Esther Delisle, The Traitor and the Jew: Anti-Semitism and Extremist Right-Wing Nationalism in Quebec from 1929 to 1939, Outremont, Robert Davies Publishing, 1993, p. 11-14. 
manière large. Très récemment, ce champ d'intérêt m'a amenée à prononcer une conférence sur le victorianisme montréalais ${ }^{14}$. Plus tard, enjambant quelques siècles avec aisance, Cook a annoté The Voyages of Jacques Cartier, c'est-à-dire la traduction par Henry P. Biggar des relations de l'explorateur. L'introduction à ce splendide ouvrage, intitulée «Donnacona Discovers Europe » et signée Cook, est en contrepoint de la vision eurocentrique des "grands explorateurs" comme Cartier et Christophe Colomb ${ }^{15}$. Cook y montre que le Canada avait sa version propre du récit des contacts à déconstruire. Il a remodelé ces idées dans un opuscule publié par le Robarts Centre sous le titre 1492 and All That: Making a Garden out of a Wilderness, dans lequel il commente la suffisance qui amena les Européens de jadis à affirmer que les Amérindiens avaient été subjugués par leurs façons de faire ${ }^{16}$. Cook semblait avoir une touche magique. Ses recherches en tout étaient méticuleuses et il théorisait ses sujets d'une manière qui en facilitait la compréhension, avec une adresse à laquelle ses étudiants ne pouvaient qu'aspirer. Le plus important pour moi, c'est que ses textes faisaient d'excellentes lectures. Ramsay Cook n'était pas de ces érudits qui, ayant découvert un champ d'études, le retournent dans tous les sens et l'exploitent pendant toute leur carrière. Ce qui le distingue, outre la qualité intrinsèque de son savoir, c'est la variété des domaines auxquels il s'est intéressé. Il s'est penché, entre autres, sur la politique de

14 Sylvie Beaudreau, «Re-imagining London in a North American Context: PsychoGeographic Spaces and Urban Tropes in Victorian Montreal ", exposé présenté au colloque annuel de la Literary London Society, "London and the Globe ", Londres, 6 au 8 juillet 2016.

15 Ramsay Cook (dir.) et Henry Percival Biggar (trad. et éd.), The Voyages of Jacques Cartier, Toronto, University of Toronto Press, 1993.

16 Ramsay Cook, 1492 and All That: Making a Garden out of a Wilderness, Toronto, Robarts Centre for Canadian Studies, 1993. Autre signe de la culture et du sens de l'humour de Cook, même sur des sujets sérieux, son titre s'inspire sans doute de la conférence de son ancien directeur de thèse, Donald Creighton, «1867 and All That ", prononcée en mars 1966 à l'Université de Toronto. Et les deux professeurs s'inspirent certainement du pastiche de l'histoire anglaise de W. C. Sellar et de R. J. Yeatman, 1066 and All That: A Memorable History of England (1930). 
J. W. Dafoe, le nationalisme québécois, le nationalisme canadienanglais, l'autonomie des provinces, l'identité occidentale, le concept de frontière au Canada, l'histoire des femmes, la critique sociale canadienne à la fin de l'époque victorienne, la littérature sur les contacts entre Européens et Amérindiens, les jardins et les artistes ayant exploré une certaine vision du Canada, dont William Kurelek et Cornelius Krieghoff. C'est un splendide héritage qui, je crois, m’a incitée de même à travailler sur une multitude de sujets. Il est vrai que l'histoire du Canada est un domaine qui, comme tous les champs d'études universitaires, s'est de beaucoup élargi au cours de la seconde moitié du $\mathrm{xx}^{\mathrm{e}}$ siècle, au point qu'il est de plus en plus difficile à une seule personne de tout maitriser. On peut toutefois constater que les esprits érudits comme celui de Cook, capables d'embrasser une telle variété de domaines, se sont raréfiés à notre époque d'hyperspécialisation et de cloisonnement. C'est peut-être une façon de dire que Cook était un « homme de la Renaissance » du Canada anglais, un concept usé, peut-être, mais qui n'en est pas moins pertinent.

Sur le plan personnel, Cook a été une source d'inspiration pour ses doctorants. Ceux qui ont eu le plaisir d'être de ses amis ont découvert que sa femme était une professeure de littérature très estimée, qu'il était collectionneur d'art, adepte de la natation et fervent ornithologue. Il attendait beaucoup de tout, s'impatientait souvent des travaux laissés en plan ou mal faits et pouvait même fulminer si les choses n'allaient pas comme prévu. C'était, par ailleurs, un homme férocement loyal, qui s'occupait très bien de ses étudiants, appréciait les bons mots et les conversations sans fin. J'ai parfois eu l'impression que l'admiration de ses étudiants lui paraissait immodérée. À l'occasion de fêtes au département d'histoire et d'activités sociales dans le cadre de colloques universitaires, il semblait souhaiter que les étudiants le traitent comme l'un d'eux. Malgré sa grande réputation d'intellectuel, Cook restait jeune de cœur. Quittant le bar de l'hôtel après quelques verres de fin de soirée avec un groupe de l'Université York, lors d'un congrès de la Société historique du Canada à Windsor, en Ontario, nous sommes passés devant une série de téléphones. Ramsay a soulevé chaque combiné pour le reposer sur 
l'appareil voisin. Oui, c'était également un farceur, ce qui en a fait une sorte d'idole pour ceux d'entre nous qui pouvaient tout juste prétendre au titre d'acolytes.

\section{Témoignage de Xavier Gélinas}

J'ai fait la rencontre tant attendue de Ramsay Cook à la fin de 1990, peu après mon arrivée au programme doctoral en histoire de l'Université York. Le Montréalais de naissance que je suis souhaitait prendre un peu de distance à l'égard du Québec et de ses débats politiques, universitaires et médiatiques tournant obsessionnellement autour de la «question nationale ». Comme la professeure Beaudreau le rappelle, le climat de l'époque était accaparé par l'accord du lac Meech, ses tenants et aboutissants, ses laudateurs et vitupérateurs (tant dans le camp souverainiste pur et dur que chez les jusqu'auboutistes fédéralistes à la Trudeau). Et pourtant, je comptais poursuivre et élargir mes recherches sur le Québec, spécifiquement sur la droite intellectuelle durant la Révolution tranquille, recherches entreprises sous l'égide du professeur Pierre Trépanier, à l'Université de Montréal. Je me suis donc inscrit à l'Université York avec la ferme intention - que dis-je, la certitude - de rédiger ma thèse sous la supervision du professeur Cook. Il a suffi d'une conversation de corridor pour que ce dernier accepte de prendre sous son aile un étudiant qu'il ne connaissait ni d'Êve ni d'Adam, alors qu'il aurait très bien pu plaider le manque de temps : il se préparait à mettre fin à sa carrière à York et avait accepté, en 1989, de diriger le Dictionnaire biographique du Canada.

Disponibilité, générosité, telles ont été mes premières impressions de Cook, impressions qui n'ont fait que se maintenir et croître durant les dix longues années qu’ont requis le programme de séminaires, puis la gestation de la thèse ${ }^{17}$. Humanité, aussi : Cook comprenait que les thésards ne sont pas des robots programmés pour produire à

${ }^{17}$ La thèse s'est muée en livre en 2007 : Xavier Gélinas, La droite intellectuelle québécoise et la Révolution tranquille, Québec, Les Presses de l'Université Laval, 2007, coll. «Cultures québécoises ». 
une cadence métronomique des chapitres successifs jusqu'à ce que l'assemblage s'appelle « $\mathrm{Ph}$. D. ». La vie des doctorants est aussi faite d'événements imprévus, heureux et malheureux, de circonstances propices ou non à l'atteinte de l'objectif, et mon superviseur comprenait cela instinctivement, parfois explicitement s'il le fallait. Dirat-on jamais assez l'importance que revêt un directeur de thèse dans la vie de son étudiant en rédaction, qui vit dans un monde insolite dans la vingtaine avancée, ni au travail comme ses contemporains ni dans le cocon sécurisant des salles de cours et des bibliothèques comme ses cadets fréquentant l'université chaque jour? Et patience, enfin. Quelle patience! Tout au long d'un échéancier qui a dû lui sembler s'allonger comme un double marathon, Cook recevait les chapitres lentement égrenés et renvoyait ses commentaires détaillés et constructifs, sans faille, dans les trente jours.

Enfin, il y avait chez Cook dans ses rapports maître-élève, de la ténacité, parfois même de l'entêtement, pour des idées qui lui étaient chères, ténacité mâtinée d'une tolérance qui finissait toujours par l'emporter. Cela n'a pas dû être facile pour un chercheur qui, certes, connaissait intimement l'histoire du nationalisme québécois et de la pensée catholique traditionnelle, mais se positionnait ailleurs sur le plan doctrinal, de devoir lire des centaines de pages abordant ces sujets avec empathie! Il faut dire que Cook se définissait lui-même comme un "unredeemable secular liberal » et que son opposition de principe au nationalisme (mais non au fait français, bien au contraire) semblait s'être raidie avec les années, comme en témoigne son essaichoc, "Alice in Meechland ", contre la clause de la société distincte dans l'accord du lac Meech ${ }^{18}$. Il ne se privait pas d'exprimer ses désaccords sur telle ou telle analyse ou telle ou telle conclusion de la thèse. Une de ses premières lettres, qui commentaient mes chapitres, se terminait loyalement par : "A good thesis deserves a good argument!

18 Ramsay Cook, "Alice in Meechland or the Concept of Quebec as a "Distinct Society" ", Queen's Quarterly, vol. 94, n 4 (1987), p. 817-828. 
So we - and especially you - are off to a good start $^{19}$ ". Mes chapitres finissaient par recevoir l'aval du maître, cependant. Cook reconnaissait, chez sa tête de mule d'étudiant, une sincérité et un certain sérieux, qualités qu'il prisait. Me permettra-t-on de citer de longs extraits d'une lettre qui résume bien son attitude?

Thank you for your letter which, as always, demonstrates your seriousness of intellectual purpose and your tolerance of your supervisor's "liberalism". The fact that you chose to work with me - though the choice at York was not that great! - assured me that you were interested in scholarship not propaganda. I hope you thought that my interest was the same. I accept that neither of us can be completely objective, but that both of us should do our best to reach that ideal.

$[\ldots]$

I have always taken the view that the final version of a thesis is intended to express the student's interpretation, not that of the supervisor. My role is to ask questions in an effort to make certain that you do mean what you are saying, that what you say is well-documented and that you will be able to defend your conclusions when the work comes up for examination. But it is your thesis and you must defend it. Of course if you cannot do so part of the reason will be that I have not done my job of forcing you to defend it to me first $t^{20}$.

Ne rêverait-on pas tous d'un superviseur doctoral qui, après avoir fait son travail de critique - travail qui a indubitablement conduit à maintes améliorations et corrections de ma part - laisse ainsi la bride sur le cou de son poulain, reconnaissant que le vrai mentorat ne consiste pas à former des clones, mais des chercheurs qui seront, à leur tour, responsables de leurs idées, pourvu qu'elles soient présentées selon les règles de l'art? Le professeur Cook incarnait le libéralisme à son meilleur. Cette tolérance authentique, cette pleine acceptation

19 Lettre de Ramsay Cook à Xavier Gélinas, 30 septembre 1996, communication personnelle.

20 Lettre de Ramsay Cook à Xavier Gélinas, 28 avril 1999, communication personnelle. 
de l'expression de vues qui n'étaient pas toujours conformes aux siennes, je l'ai vue en action, après la fin de ma thèse, chez Ramsay (il nous conviait - il l'exigeait même - à l'appeler par son prénom, sitôt le diplôme obtenu). Même s'il était entièrement dégagé, tant moralement qu'administrativement, de sa tâche de conseiller et d'encourager son étudiant, j'entendais, par personnes interposées, les bons mots qu'il voulait bien prodiguer à mon égard auprès de collègues, d'institutions, de mon employeur... C'est par la reconnaissance que je désire clore ce bref témoignage.

\section{Pour une étude approfondie}

Nous n'avons eu d'autre ambition que de présenter rapidement Ramsay Cook et de témoigner notre dette à l'égard d'un maître qui n'était pas sans défaut, certes, mais dont la bienveillance, tant intellectuelle que personnelle, la curiosité d'esprit et la productivité inlassable pèsent lourdement et favorablement dans la balance. La place considérable de Cook dans l'historiographie exigerait une étude en profondeur, du genre de celles qu'ont produites Jean Lamarre sur l'École de Montréal des Brunet, Frégault et Séguin, Carl Berger sur les historiens anglo-canadiens ou Donald Wright sur le superviseur même de Cook, Creighton, tout récemment. Le professeur Wright s'est justement attelé à cette tâche et a déjà livré quelques aperçus prometteurs ${ }^{21}$. Souhaitons que la biographie méritée voie le jour sans tarder. Au-delà de l'éclairage d'une œuvre de premier plan, pareille étude ouvrira une fenêtre sur de vastes pans de l'histoire de la vie intellectuelle canadienne de la seconde moitié du $\mathrm{xx}^{\mathrm{e}}$ siècle.

${ }^{21}$ Par exemple, voir Donald Wright, "Introduction to the Second Edition ", dans Cook, The Regenerators, p. xxiii-xlviii ; "Sesquicentennial Cerebrations ", Acadiensis, vol. XLVI, n 1 (hiver-printemps 2017), p. 145-151; et "Ceci n'est pas Ramsay Cook: A Biographical Reconnaissance ", dans Doug Munro et John G. Reid (dir.), Clio's Lives: Biographies and Autobiographies of Historians, Acton, Australian National University Press, 2017, p. 103-134, qui présente un regard fascinant sur les années de formation de Cook. Nous remercions le professeur Wright de nos échanges lors de la préparation de cet article. 